\title{
Point Me In the Right Direction: Understanding User Behaviour with As-The-Crow-Flies Navigation
}

\author{
Gian-Luca Savino \\ University of Bremen \\ Bremen, Germany \\ gsavino@uni-bremen.de
}

\author{
Laura Meyer \\ University of Bremen \\ Bremen, Germany \\ lameyer@uni-bremen.de
}

\author{
Eve Emily Sophie Schade \\ University of Bremen \\ Bremen, Germany \\ eve.s@uni-bremen.de
}

\author{
Thora Tenbrink \\ Bangor University \\ Bangor, UK \\ t.tenbrink@bangor.ac.uk
}

\begin{abstract}
Visual as-the-crow-flies (ATCF) navigation methods are an increasingly popular alternative to existing turn-by-turn (TBT) navigation for cyclists. To better understand how people use them in everyday navigation and how they cope with the novel navigation method in challenging situations, we studied two main issues posed by ATCF navigation: knowing whether one is on the right route to their destination and knowing whether a turn leads into a dead end or detour. To investigate these two problems, we compared visual ATCF navigation against (1) TBT navigation and (2) an improved $\mathrm{ATCF}+$ navigation system in two successive studies. We found that users encountered problems riding in the opposite direction to the destination and were often turning around as a result using the ATCF method. Using colour cues in the ATCF user interface we were able to reinforce correct route choices. Additionally, we found that unsuccessful route progression negatively correlates with user confidence.
\end{abstract}

\section{CCS CONCEPTS}

- Human-centered computing $\rightarrow$ Human computer interaction (HCI); User studies; Field studies; Empirical studies in ubiquitous and mobile computing.

\section{KEYWORDS}

As-The-Crow-Flies; Navigation; Bicycle; Wayfinding; Turn-By-Turn; Field-Study; Mobile

\section{ACM Reference Format:}

Gian-Luca Savino, Laura Meyer, Eve Emily Sophie Schade, Thora Tenbrink, and Johannes Schöning. 2020. Point Me In the Right Direction: Understanding User Behaviour with As-The-Crow-Flies Navigation. In 22nd International Conference on Human-Computer Interaction with Mobile Devices and Services (MobileHCI '20), October 5-8, 2020, Oldenburg, Germany. ACM, New York, NY, USA, 11 pages. https://doi.org/10.1145/3379503.3403539

Permission to make digital or hard copies of all or part of this work for personal or classroom use is granted without fee provided that copies are not made or distributed for profit or commercial advantage and that copies bear this notice and the full citation on the first page. Copyrights for components of this work owned by others than the author(s) must be honored. Abstracting with credit is permitted. To copy otherwise, or republish, to post on servers or to redistribute to lists, requires prior specific permission and/or a fee. Request permissions from permissions@acm.org.

MobileHCI '20, October 5-8, 2020, Oldenburg, Germany

(c) 2020 Copyright held by the owner/author(s). Publication rights licensed to ACM. ACM ISBN 978-1-4503-7516-0/20/10 ..\$15.00

https://doi.org/10.1145/3379503.3403539
Johannes Schöning

University of Bremen

Bremen, Germany

schoening@uni-bremen.de
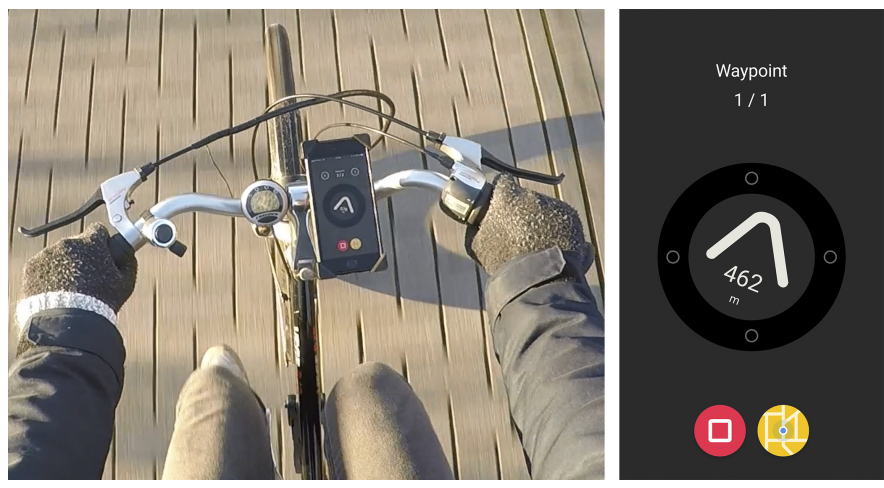

Figure 1: Beeline is a mobile navigation application with a simplistic interface. It consists of a single arrow pointing into the straight line direction of the next waypoint or destination and additionally displays the straight line distance to them. () Beeline.

\section{INTRODUCTION \& MOTIVATION}

Turn-by-turn (TBT) guidance is the default navigation method for most kinds of transportation modes, including bicycles. Its ability to guide people from A to B along a shortest or fastest path, using clear instructions about where to turn at each decision point, has been proven to be the preferred routing choice for most people already back in 1995 [10]. And in spite of many research studies that investigate alternative routing options like safest, simplest or most scenic [8, 17, 28, 35], the fastest route remains the default option in many car navigation systems more than twenty years later. In contrast to that, we now see novel navigation methods called "compass mode" or "as-the-crow-flies" being introduced into the commercial domain of bicycle navigation.

The as-the-crow-flies (ATCF) navigation method, while novel for most users, is not unknown to the research community. Instead of giving TBT instructions to guide the user, it indicates the general direction to the destination. It is a sub domain of mobile navigation research, and has its origins in the development of navigation methods for the blind [19].

When looking at bicycle navigation research many studies revolve around making navigation less intrusive, allowing cyclists to focus more on their surroundings as cycling in heavy traffic 
is not only demanding but also potentially dangerous [11, 18, 31] This is often achieved by using auditory $[1,12,16,20,36]$ or tactile $[20,25,27,33]$ cues to convey the navigation instructions. Other modalities include instructions conveyed through light signals inside the helmet [20,34], on an external navigation device [7] or projections in front of the bicycle [5, 6]. Most of these navigation systems use TBT navigation, giving information on where to turn at each decision point. Some of them do use ATCF navigation, but only in combination with auditory or tactile cues. However, commercially available products $[2,4,32]$ exclusively use visual user interfaces. The Beeline application shown in figure 1 is one example and was used in our studies. The interface features an arrow that indicates the direction of the destination or the next waypoint and information about the remaining distance.

Based on related literature by Hochmair [13] and Albrecht et al. [1], we identified two main challenges that confront users of ATCF navigation: (1) knowing whether they are currently on a route leading to their destination; (2) knowing whether a potential turn might result in a dead end or detour.

We studied these challenges of everyday use of visual ATCF navigation methods and by doing so contribute to the understanding of ATCF navigation. We conducted two studies:

- Study 1 compares a commercial implementation of ATCF with a commercial implementation of TBT navigation.

- Study 2 takes the insights of study 1 to build an improved version of the implementation and compares two ATCF navigation interfaces.

Both studies use an A/B testing design allowing us to observe user behaviour related to the above mentioned challenges. We present quantitative data recorded from participants' performances (route length, number of errors, task load and orientation) and give insights about wayfinding and users' navigation behaviour with ATCF navigation based on observations and interviews.

Our results show that whenever the destination lies in the opposite direction to the one in which the user is currently riding, users respond by either turning around or trying to find a different route, even if that means carrying their bike up a staircase. In order to travel strictly in the direction provided by the navigation system some participants even rode through private property. We derive and evaluate design implications to counter these reactions and improve users' confidence while using ATCF navigation.

In line with previous literature [1] we find that a basic implementation of ATCF navigation, as it is used in most commercial products, can have major disadvantages compared to TBT navigation when looking at navigation efficiency (reaching a destination as fast as possible without errors), at least with a route design like the one we used in our study. To explain these disadvantages we report on several situations that proved a challenge for ATCF navigation and test how much, if at all, these same situations affect TBT navigation. We present indicators that ATCF navigation can lead to improved orientation abilities, as participants had better knowledge of the direction of their initial starting position compared to TBT users. Furthermore, we confirm findings from Pielot et al. [25] that participants liked the ATCF navigation method for leisure biking.

Additionally, we identify that communicating progress to the destination during the navigation task is a very crucial aspect of building confidence. In our implementation this was communicated through the remaining straight line distance to the destination on the top half of the screen (see figure 4a). However, this distance increased whenever participants were getting further away from the destination, which (apart from errors) naturally happened when they had to find a way around some obstacle (see figure 6a and 6b). Being used to TBT navigation, where the distance to the destination continuously decreases, many participants associated the rising distance with negative feedback. This clearly impacted users' confidence about whether they were still on the right track.

\section{RELATED WORK}

This section summarises previous research relevant to ATCF navigation. We present the underlying wayfinding heuristic "Least Angle Strategy" (LAS), followed by related research on ATCF in combination with auditory and tactile cues $[1,16,23-26,30,36]$.

\subsection{Least Angle Strategy}

The heuristic underneath the ATCF navigation method was first formulated by Hochmair in 2000 [14] and is called the Least Angle Strategy: "At the beginning of the navigation, the agent perceives his navigation target [...]. He moves in a street network and navigates towards the target without using a paper map. At an intersection, he takes the road which has the least deviation $\alpha$ from the direct target direction. In a city, physical objects often block the direct view to the target. Then [...] estimations of directions and distances, especially of a reference direction, need to be made. The estimations can be disturbed by estimation errors" [14].

ATCF navigation aids are designed to eliminate these estimation errors. Instead of keeping track of the direction towards the destination, which can be challenging after a few turns with no landmark to orient towards [15], one can make use of the constant direction indication for each navigation decision. This way the LAS can always be used to its maximum potential. Based on this description of the LAS and the way visual ATCF navigation like Beeline works, the following scenarios may occur in which people are likely to run into problems using LAS with ATCF navigation:

(1) The user is generally uncertain whether the current route is progressing towards the destination.

(2) There is a street that points in the direction of the destination, but the user is unable to see its end, so it might be a dead end or turn away from the destination.

(3) Maybe as a result of previous situations, the arrow is facing in the opposite direction to the direction of travel (i.e., the destination is currently behind the user), and there is no immediate way to turn other than making a U-turn.

To better understand how users might deal with such scenarios, we look at related work addressing ATCF navigation methods with auditory or tactile cues.

\subsection{Direction Conveyed through Sound}

The idea of using spatial audio for navigation revolves around humans' ability to localise the source of sound [3]. In navigation this was initially explored by Loomis et al. (1998) [19] to build assistive navigation technology for the blind. They used spatial audio successfully to guide blind people along a predefined path in 
an open field environment. A few years later, Holland et al. (2002) built a first mobile version using spatial audio for pedestrians and car drivers. While they found that it was usable for locating targets on foot, it was insufficiently responsive to be used in a car [16].

The first time this approach was successfully used on a bicycle was in a study on spatial audio for beacon (ATCF) or route (TBT) guidance by Albrecht et al. [1]. They were able to route pedestrians and cyclists through a suburban environment using spatial audio in an ATCF manner. They used spatial audio to indicate the direction towards the destination and volume to present the distance between the user and the destination. In general they found that beacon guidance (ATCF) was preferred for more familiar surroundings and touristic exploration. Route guidance (TBT) was considered the better alternative for people in a hurry [1]. In one condition they found that participants experienced uncertainty walking on a straight path with nowhere to turn toward the direction of the destination, as there was no way of knowing whether that street would eventually lead to the destination. This supports our intuition (cf. situations 1 and 2 above) that such scenarios are problematic for ATCF navigation users.

\subsection{Direction Conveyed through Vibration}

Auditory cues rely on a sense that is also used to identify possible threats in traffic. In contrast, the sense of touch is not otherwise required and thus available for input during cycling. This makes vibration an attractive choice for conveying navigation information.

Prasad et al. [27] created HaptiMotor, a vest for motor cyclists with multiple vibration motors that were able to indicate left, right, straight and U-turn at an approaching decision point. They show that HaptiMotor increases riders' situational awareness and that users found the mapping to be intuitive. A similar approach for cyclists is illustrated by Steltenpohl and Bouwer with Vibrobelt [33]. The tactile belt is worn around the waist and "gives waypoint, distance and endpoint information using directional tactile cues" [33] They compared it to a visual route guidance system and found that participants using Vibrobelt had improved spatial knowledge acquisition. These two setups require the user to wear a dedicated piece of technology to receive the directional information.

A TBT-system built directly into the bicycle was used by Matviienko et al., who compared auditory, tactile and visual TBT instructions for child cyclists [20]. Auditory navigation was the most preferred, but light- and tactile navigation cues were also positively perceived and found to be valid supplementary navigation aids for younger children. In their setup, the vibration was built into the handlebar of the bicycles. This made for a very straight forward mapping for TBT instructions. However, the same approach was also used to convey directional information for ATCF navigation in a study by Pielot et al. (2012) [25]. Instead of just having the left or right handle vibrate, the relative intensity of the vibration was used to cue the direction in relation to the bicycle's heading. They found that the cues were understandable and effective and that none of the participants lost orientation as long as haptic feedback was provided. One issue they identified was that people who were very familiar with TBT navigation still expected the system to give instructions at every turn despite very detailed instructions about the functionality of ATCF navigation. The study took place on a small, touristy, island. There were many alternative paths and the whole area was geographically constrained. "Thus, [...] participants would usually find a way to their destination as long as they kept going roughly into the right direction, and they did not have to be afraid of getting lost by going into the wrong direction for too long" [25]. The results might, therefore, not be completely transferable to sub-urban city environments, where a wrong turn can sometimes lead to large detours and traffic is a more prominent factor while navigating. The authors concluded that ATCF navigation conveyed through vibration patterns works well for longer tours in unfamiliar environments [25].

\subsection{Contribution}

In this paper we extend the related research in two main directions: First, to our knowledge there is no previous research on the usability and performance of visual ATCF navigation methods. As ATCF navigation methods steadily increase in popularity, a better understanding of their performance is paramount for guiding their development from an HCI perspective. Secondly, little is known about user behaviour while using ATCF navigation methods in contrast to TBT navigation. Most existing studies show that ATCF navigation can be used to successfully guide people from $\mathrm{A}$ to $\mathrm{B}$. However, they deliver only limited results concerning everyday problems people might face in urban environments, like running into dead ends and knowing whether or not one is still on the right track. In order to create an experimental setup that allows to address both these issues we embedded the navigational task of riding in the opposite direction to the arrow into two user studies. The first one compared ATCF to TBT on two consecutive routes. The second one improves the ATCF navigation interface to include information about dead ends, detours and correct route choices.

\section{STUDY 1 - TBT VS ATCF}

The goal of study 1 was to evaluate the differences between visual TBT and visual ATCF navigation to highlight their effect on navigation performance, orientation and how both these systems deal with the aforementioned problem scenarios, focusing particularly on the arguably most disturbing situation (3) in which users find themselves riding in the opposite direction to the arrow. Participants used Google Maps as a representative TBT navigation system and Beeline as a representative ATCF navigation system respectively on two consecutive routes. Besides observing their navigation behaviour, we tested the following hypotheses:

H1: Given our route design, participants using TBT navigation will make fewer errors and produce a shorter route than participants using ATCF navigation.

H2: Participants using TBT navigation will have poorer orientation with respect to the direction of the initial starting position than participants using ATCF navigation.

\subsection{Task \& Procedure}

Each participant cycled two different routes (see figure 2). Each started on route A and was randomly assigned a navigation method to start with, followed by the other method on route B. Thus, half of the participants used TBT navigation on route A and ATCF navigation on route $B$ (condition 1), and the other half used ATCF 


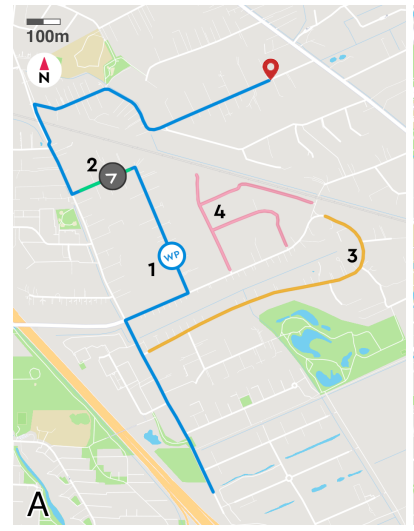

(a)

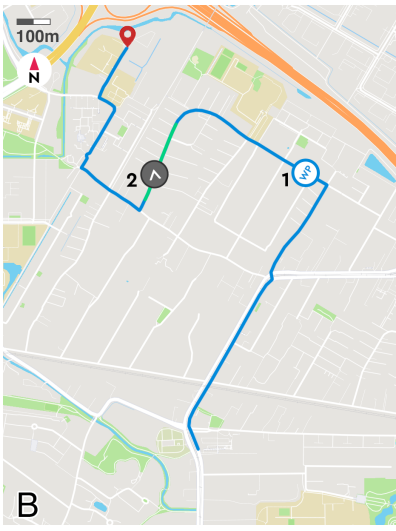

(b)
Figure 2: (left) Route A used in study $1+2$, (right) Route B used only in study 1. Map annotations: (1) Waypoint; (2) Situations where the arrow points in the opposite direction; (3) Detour participants took in study 2 with ATCF+; (4) Dead end/detour area which caused problems for participants using ATCF. Basemap $\odot$ Mapbox $\odot$ OpenStreetMap.

navigation on route A and TBT navigation on route B (condition 2). Both routes were $2.45 \mathrm{~km}$ long, had roughly 20 decision points (not counting private driveways) and low to medium traffic. Both were designed to include a part where participants would need to cycle in the opposite direction to the arrow (see figure 2, number 2). To ensure that all participants would be routed into this situation, a waypoint was added to each of the routes (see figure 2, number 1). Participants were not shown a map in advance. For safety reasons and to reduce stress during the study, we explicitly told every participant that they were free to do the task at their own pace.

Before each condition, participants were introduced to the navigation method and application they were about to use (ATCF or TBT). They were explicitly shown how the arrow behaves and functions and could ask questions. After filling in a demographic questionnaire, participants cycled the routes on their own and met the experimenters again at the destinations. After each route, an in-between questionnaire about the route and experience with the navigation device, a NASA TLX questionnaire and an orientation test (pointing into the direction of the starting location) were conducted. The participants were questioned in a semi-structured interview about the navigation method they had just used, and were asked to express their opinion and describe their experience. At the end of the study, participants were asked to describe the difference between the navigation methods, and state which one they preferred and give further comments. The semi-structured interviews were loosely transcribed to capture their content, and evaluated.

\subsection{Participants \& Apparatus}

We used a within-subjects design with 12 participants ( 9 male and 3 female, all university students) between the age of 23 and 29 $($ mean $=25.15$, median $=25, \mathrm{sd}=1.83)$. Most of them ride bikes on a regular basis and all participants had used Google Maps before but never the Beeline application (or any other kind of ATCF navigation service). Participants were asked to rate whether they were familiar with the study location on a scale from 1 (very familiar) to 6 (not familiar at all) and gave a mean rating of 4.79 (median=5, $\mathrm{sd}=1.37$ ). Google Maps and Beeline were installed on an iPhone 6s, which was mounted to the handlebar of the bicycle (see figure 1). The questionnaires were completed by the participants on an iPhone 7 . Participants additionally received a Google Pixel 2 which logged the GPS position throughout the study. All of them had overcast weather conditions.

\subsection{Measures}

Completion time was recorded but not further considered as there are too many influential factors (e.g. traffic) unrelated to the conditions. Also, participants had been told to take as much time as they liked. For purposes of analysis we used the following definitions:

Dead end: A street with only one inlet or outlet.

Detour: A street segment that causes a longer route compared to the shortest path from the participants' position to the current destination.

The following five factors were measured.

3.3.1 Route length. The actual route participants took and its length was calculated from the GPS trace of their position (see figure 3). Even with our best efforts to filter and clean the data, the GPS trace included variations unrelated to participants' decision making. Therefore we retraced the routes using openrouteservice [22].

3.3.2 Errors. An error was defined as an event where participants turned around after noticing or thinking that they had gone the wrong way, and returned using the exact same route that was used to enter the street (e.g. running into a dead end).

3.3.3 Task Load. The task load was measured using NASA TLX through the NASA TLX application provided by NASA [21].

3.3.4 Orientation. After each condition, participants were asked to point in the direction of the starting position using the native compass app of a freshly calibrated iPhone $6 \mathrm{~s}$. We calculated the deviation to the correct angle.

3.3.5 Wayfinding Behaviour. In addition to the quantitative measures above we used our observations, questionnaires and interviews to understand and analyse participants' behaviour, including the situation where they had to ride in the opposite direction to the arrow.

\subsection{Results}

All participants were able to complete both routes successfully. The following results are presented for each of the two routes separately. We used Welch Two Sample t-tests for normally distributed data (indicated by reported $\mathrm{t}$-value) and Wilcoxon rank sum tests for not normally distributed data (indicated by reported $\mathrm{W}$-value). To ensure that familiarity with the environment did not influence the results, we tested for correlations between our measures and familiarity. No correlation was found for any of them $(p>0.05)$.

3.4.1 Route Length. As expected, participants produced significantly shorter routes using TBT navigation in comparison with 


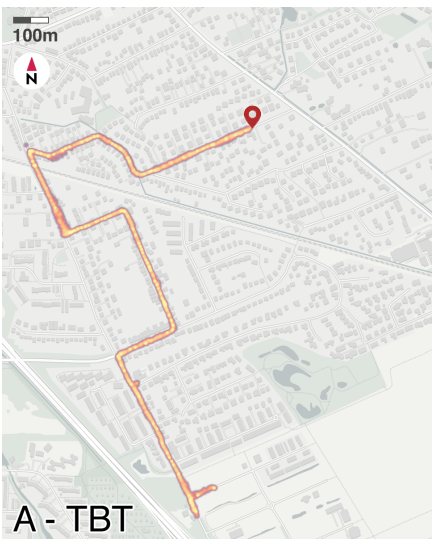

(a)

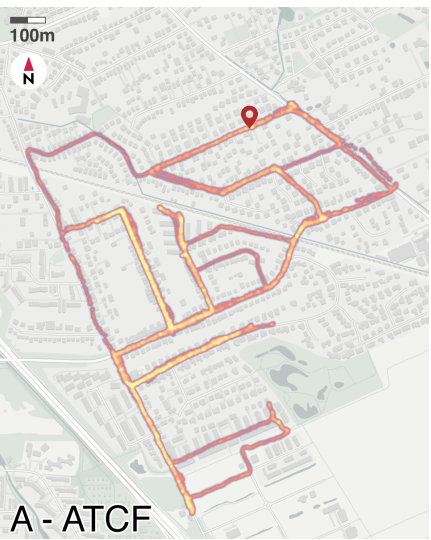

(b)

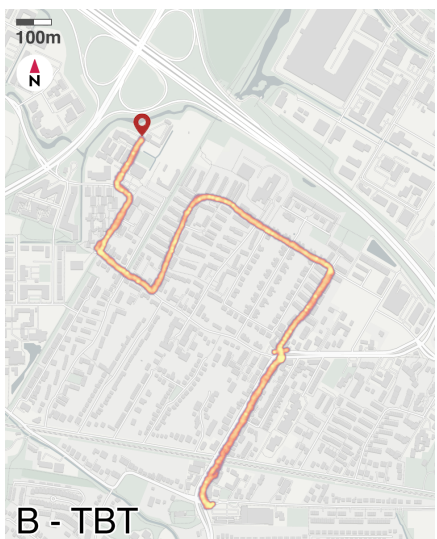

(c)

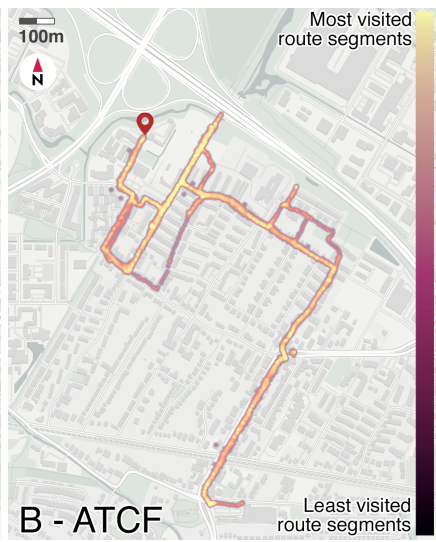

(d)

Figure 3: Heat maps of the uncorrected location data of all participants from the respective combinations of routes and navigation methods in study 1 . Basemap $\odot$ OpenStreetMap.

ATCF navigation. Because our TBT application was set to calculate the shortest path, even a small mistake or detour using ATCF immediately means a longer route. On route A, TBT based routes (mean $=2467 \mathrm{~m}$, median $=2450 \mathrm{~m}, \mathrm{sd}=41 \mathrm{~m})$ were significantly shorter $(\mathrm{W}$ $=0, \mathrm{p}=0.0037 \mid$ effect size $=0.86, \mathrm{ci}=0.64-0.94)$ than ATCF based routes $($ mean $=4228 \mathrm{~m}$, median $=4282 \mathrm{~m}$, $\mathrm{sd}=583 \mathrm{~m})$. Likewise, on route B, TBT based routes $($ mean $=2450 \mathrm{~m}$, median $=2450 \mathrm{~m}, \mathrm{sd}=$ $0 m)$ were significantly shorter $(\mathrm{W}=0, \mathrm{p}=0.0028 \mid$ effect size $=0.89$, $\mathrm{ci}=0.73-0.95)$ than ATCF based routes $($ mean $=3076 \mathrm{~m}$, median $=$ $2882 m, \mathrm{sd}=532 \mathrm{~m})$.

3.4.2 Errors \& Riding In the Opposite Direction to the Arrow. On route A participants made a total of 14 errors using ATCF navigation compared to 1 error using TBT navigation. On route B participants made a total of 9 errors using ATCF navigation compared to 0 errors using TBT navigation. Most of the errors on route A included driving into a dead end whereas on route $B$ the most frequent error was riding in the wrong direction with no way to correct but to turn around.

8 out of the 24 total errors were caused by participants having to ride in the opposite direction to the arrow. This happened to 4 participants on route A who turned around afterwards and to 4 participants on route B who found another route that led toward the target direction. This route required them to carry their bike up the stairs which they did despite of the instruction to not get off their bike during the ride. None of this behaviour was shown by any of the participants using TBT navigation on either route.

3.4.3 Task Load. The task load was significantly lower for participants using TBT navigation on both routes. On route A the participants' task load using TBT navigation had a mean value of $36.7($ median $=34.5, \mathrm{sd}=14.5)$ compared to $55.8($ median $=54.3$, $\mathrm{sd}=12.5)$ with ATCF navigation $(\mathrm{t}=-2.46, \mathrm{df}=9.8, \mathrm{p}=0.0344$ effect size $=1.42$, $\mathrm{ci}=0.1-2.68)$. On route $\mathrm{B}$ their task load using TBT navigation had a mean value of $16.3($ median $=13.5, \mathrm{sd}=10.3)$ compared to 47.7 (median $=49.5$, $\mathrm{sd}=21.5$ ) with ATCF navigation $(\mathrm{t}=-3.23, \mathrm{df}=7.16, \mathrm{p}=0.0141 \mid$ effect size $=1.86, \mathrm{ci}=0.44-3.22)$.
3.4.4 Orientation. Numerically, participants using ATCF navigation appeared to perform better in estimating the direction of the starting location on both routes. On route A results showed a higher mean deviation angle $(\mathrm{W}=21, \mathrm{p}=0.6991 \mid$ effect size $=-0.14, \mathrm{ci}=$ $-0.74-0.47)$ for $\mathrm{TBT}\left(\right.$ mean $=48^{\circ}$, median $\left.=42^{\circ}, \mathrm{sd}=28^{\circ}\right)$ than for ATCF $\left(\right.$ mean $=35^{\circ}$, median $\left.=32.5^{\circ}, \mathrm{sd}=13^{\circ}\right)$. Likewise, on route B results showed a higher mean deviation angle $(\mathrm{t}=1.1 \mathrm{df}=6.16, \mathrm{p}$ $=0.3109 \mid$ effect size $=0.64, \mathrm{ci}=-0.54-1.79)$ for TBT $\left(\right.$ mean $=53^{\circ}$, median $\left.=28^{\circ}, \mathrm{sd}=59^{\circ}\right)$ than for $\operatorname{ATCF}\left(\right.$ mean $=25^{\circ}$, median $=22^{\circ}$, $\mathrm{sd}=20^{\circ}$ ). Although these results were not statistically significant, the lower standard deviation for ATCF could hint towards improved orientation in the environment as compared to TBT navigation.

\subsection{Summary}

Our results show that TBT navigation leads to better performance in terms of route length, errors and task load. This confirms H1. Routes with TBT navigation are shorter than with ATCF and lead to fewer errors. The difference in route length shows a large effect with medium confidence intervals. The observed effect is therefore very likely to exist. However, for task load the effect is large with a large confidence interval and the orientation results show small to medium effect sizes with large confidence intervals, which means for both more data would be needed in future work to clearly state whether we can be sure that these observed effects exist (according to thresholds presented in [29]). A post-hoc power analysis using $\mathrm{G}^{*}$ Power for study 1 revealed a mean power of 0.23 [9].

It is important to note that the route design most likely had an impact on this outcome as the situation where the arrow points in the opposite direction was expected to negatively influence the performance of ATCF navigation users. The comparison gives a first impression of how strong this influence was.

When asked whether they could have found their way back to the starting location if the navigation device had failed during their ride, 7 out of 12 participants answered yes for ATCF and 8 out of 12 answered yes for TBT. So in both conditions participants felt just as sure that they could find their way back. When asked to rate their orientation ( 1 - very well oriented; 6 - no orientation at all), 
participants using TBT gave a mean rating of 1.75 while participants using ATCF gave a mean rating of 3.08 - contrary to the results of the orientation test. A possible explanation for this could be that participants were generally less confident with the ATCF navigation method. Only one participant using ATCF was always sure to be on the right route to their destination compared to 9 participants who felt the same way using TBT. Participant 6 commented on this (translated from the German original): "When I was not going into the direction the arrow pointed in, I felt frustrated. Because I wasn't sure, in an unknown environment, whether a street would turn up that pointed in the direction of my destination, or if I should turn back and try a different street". Based on our results we are not able to statistically confirm $\mathrm{H} 2$ but see indications that ATCF might have advantages over TBT navigation when it comes to orientation.

A situation that clearly added to the insecurity of participants using ATCF navigation was having to ride in the opposite direction to the arrow. 4 out of 6 participants on both routes A and B turned around or away when faced with this situation. On route $B$, we observed just how much people tried to move in the direction of the arrow. Figure $3 \mathrm{~d}$ shows that instead of following the road and riding against the arrow for a short while, participants turned around or immediately took a small pathway that led in the target direction, even if that meant carrying their bike up a staircase (while scouting the routes, we were actually not aware of this "shortcut"). On route A there were no further options than to either carry on against the arrow's direction, or to turn around (see figure $3 b$ ) - which is what 4 out of 6 participants did. This demonstrates indicatively how users deal with difficult navigation situations using ATCF.

ATCF users also reported difficulties developing survey knowledge of the area. For many, this resulted in running into (sometimes even multiple) dead ends (see figure 3b). Beeline didn't seem to be "[...] very helpful when it came to getting an overview" (participant 6), whereas with Google Maps they "always knew the optimal streets" (participant 7).

3.5.1 Implications for Study 2. From the results of this study, we deduced the main shortcomings of a basic visual implementation of ATCF navigation. (1) There is no information about whether one is still on the right route to the destination (leading some users to turn around even when they don't have to) and (2) there is no way to get information about one's surroundings (like adjacent streets, detours or dead ends). Based on these shortcomings we built our own prototype of a more advanced visual ATCF navigation application, which we evaluate in study 2 to gain further insights into users' behaviour when using ATCF navigation.

Before describing the design of our own prototype it is important to mention that the Beeline tool itself includes a "Route Assistant" feature (not used in our study), which creates waypoints at every decision point along the route. This effectively makes it a turn-byturn approach in which ATCF navigation is only used to get to the next intersection. In contrast, our redesign aims to keep most of the ATCF functionality while enabling users to make more informed navigation decisions.

\section{STUDY 2 - ATCF VS ATCF+}

From the results of study 1 we were able to identify design implications whose effects we could test in an improved ATCF+ version.
In particular, we added three kinds of visual cues to inform the user whether they are still on the right route to their destination or heading into a dead end or detour, plus further information about their surroundings. In detail these design changes include:

- Background colour indicating whether users are on the right route to their destination (green, see figure 4b), taking a detour (yellow, see figure 4c) or heading towards a dead end (red, see figure 4d).

- Visual cues that indicate upcoming dead ends or detours (see figure 4a).

- A mini map with live updates about the users position (see figure 4).

These changes were designed to alleviate the main shortcomings of the application used in study 1 . To implement the changes we developed our own visual ATCF navigation application for Android following the example set by Beeline. This approach allowed us to have two versions of our app: one which is identical in functionality and very similar in design to the Beeline application used in study 1 , and another with the above mentioned features.

For consistency, this study has a similar setup to study 1 except for a few changes which will be described in the following subsections. The main difference is that we used only route A with a between-subjects approach, with participants in one condition using the ATCF version and participants in the other condition using the extended ATCF+ version. To identify more detailed implications we wanted to focus only on one aspect of error handling: the behaviour of turning around after having to ride in the opposite direction to the arrow. Therefore we replicated study 1 for the ATCF condition on route A, and added another condition (ATCF+) for direct comparison. We did not include route $B$ because of the unexpected staircase-based shortcut that some participants used. Our hypotheses were as follows.

H1: Participants using ATCF+ will be less likely to turn around when they have to ride in the opposite direction to the arrow than participants using ATCF navigation.

H2: Participants using ATCF+ will make fewer errors than participants using ATCF.

H3: Routes with ATCF+ will be shorter than routes with ATCF.

\subsection{Task \& Procedure}

Each participant cycled one route (route A from study 1, see figure 2a) and was randomly assigned the condition ATCF or ATCF+ (7 participants per condition). Before starting their ride they were shown the application and an info graphic explaining its functionality. For the ATCF+ condition this included the various interface changes and their meaning. As in study 1 they were not shown a map and were instructed to go at their own pace. They filled in a demographic questionnaire, cycled the route on their own and met the experimenters at the destination. As in study 1 , after reaching the destination they were asked to complete another questionnaire about the route and experience with the navigation device, a NASA TLX questionnaire and an orientation test (pointing into the direction of the starting location). Finally, participants were able to give comments in a semi-structured interview. 


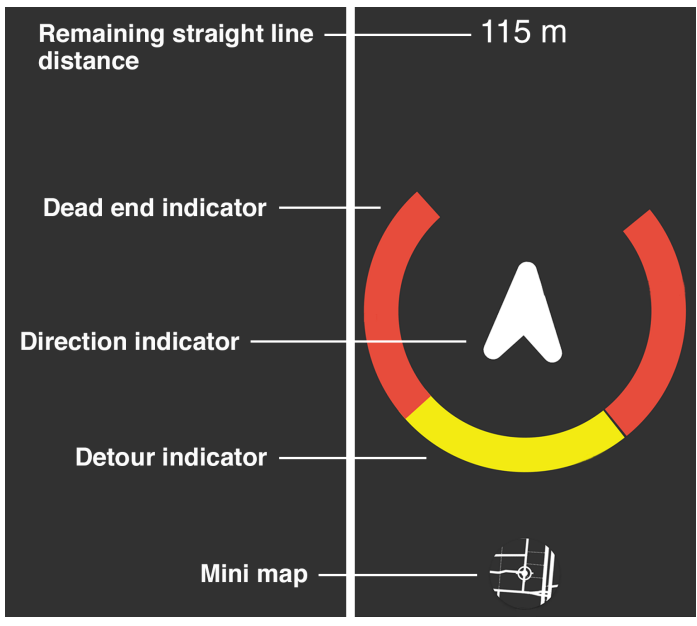

(a)

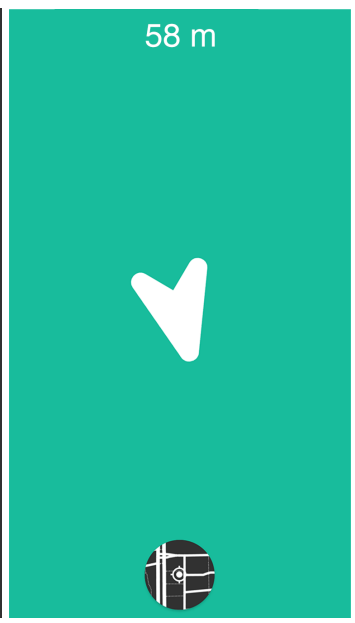

(b)

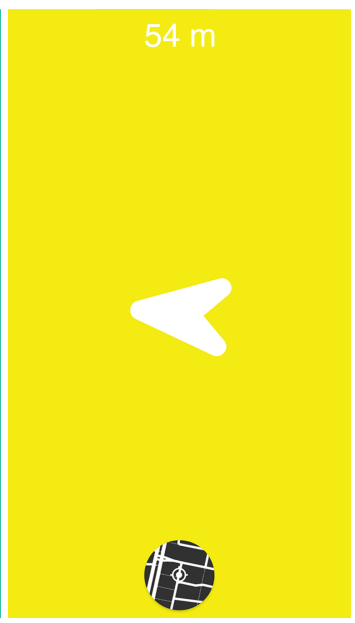

(c)

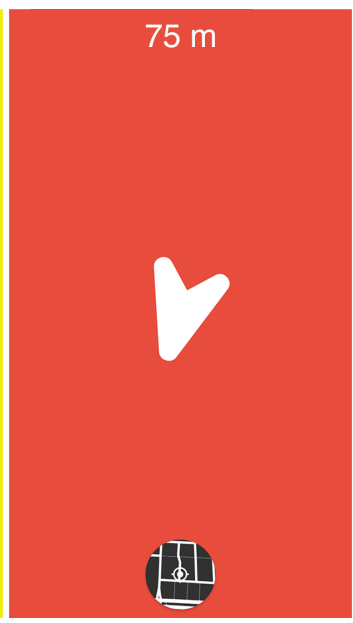

(d)

Figure 4: ATCF+ interface: (a) A detour behind the user and a dead end to the left and right; (b) The user is on the right route to their destination, even though it lies in the opposite direction; (c) The user is on a detour; (d) The user is in a dead end

\subsection{Participants \& Apparatus}

We used a between-subjects design with 14 participants (7 male and 7 female; 7 students and 7 with varying job roles) between the age of 22 and 60 (mean=30.86, median=25, sd=13.17). 10 out of 14 said they cycled frequently (three or more times a week). None had heard of or used any ATCF navigation before the study. Participants' familiarity rating with the environment $(5=$ very familiar, $1=$ not familiar at all) resulted in a mean rating of 2.5 (median=3, sd=1.02). Both the ATCF and the ATCF+ applications were installed on a Samsung Galaxy Note 8 which was mounted to the handlebar of the bicycle as in study 1 . The same smartphone was used for completing the questionnaires and tracking the GPS position using our own navigation applications. All participants had sunny to overcast weather conditions.

\subsection{Technical Implementation}

The ATCF+ application uses a graph representing the street network of the local area around route A. The graph consists of several nodes per street. Each node holds information on whether it is part of a dead end or detour. We use the GPS sensor and magnetometer of the smartphone to implement the ATCF (compass) navigation. Whenever the user gets into the vicinity $(25 \mathrm{~m})$ of a node, said node triggers an event, for example if its neighbouring node is a dead end. Using the graph and the user's location the system knows how far the user is into a dead end or detour and can react accordingly. To make sure participants would not go too far off track we added "dead end nodes" all the way around the study area.

\subsection{Measures}

The measures are identical to study 1: route length, errors, task load, orientation and wayfinding behaviour. Additionally, we evaluated the straight line distance to the destination over time.

\subsection{Results}

All participants were able to complete the route successfully with either navigation method. The following results compare both conditions, ATCF and ATCF+, to each other. To ensure a valid comparison between the sample of each study, a Wilcoxon rank sum test did not find any significant differences between both age groups $(\mathrm{W}=84.5, \mathrm{p}=1)$. As before, no correlation was found between participants' familiarity with the environment and the following test results $(\mathrm{p}>0.05)$ in study 2 .

4.5.1 Route Length. Route length was not significantly different between the two conditions $(\mathrm{t}=-1.25, \mathrm{df}=9.41, \mathrm{p}=0.2428 \mid$ effect size $=0.67, \mathrm{ci}=-0.43-1.73)$. Contrary to our expectations, it even seemed that participants using the ATCF navigation method used shorter routes $($ mean $=4163 m$, median $=4140 m$, sd $=1389 m)$ on average than those using $\mathrm{ATCF}+($ mean $=5504 \mathrm{~m}$, median $=6100 \mathrm{~m}$, $\mathrm{sd}=2486 \mathrm{~m})$.

4.5.2 Errors \& Riding In the Opposite Direction to the Arrow. Participants made almost the same number of errors with a total of 20 errors using ATCF navigation compared to 21 with ATCF+. But as figure 5 shows, participants struggled less with the dead end/detour area using ATCF+ (compare figure $5 \mathrm{a}$ and $5 \mathrm{~b}$ and see figure $2 \mathrm{a}$, number 4).

In general ATCF+ did address some of the errors caused by ATCF but it also introduced new ones: with ATCF 5 out of 7 participants turned around when they had to ride in the opposite direction to the arrow (similar to the $4 / 6$ when using ATCF in study 1 ). None showed this behaviour with ATCF+. One participant using ATCF+ did not face this situation, as they went a completely wrong way and then entered the street from the other side (see figure 2a, number 2). The remaining $6(\mathrm{ATCF}+)$ participants all got to the end of the road successfully after the first waypoint.

The following T-intersection unveiled another problem caused by the functionality of ATCF navigation: Whenever the arrow faces either straight ahead or straight back at an intersection, users have 


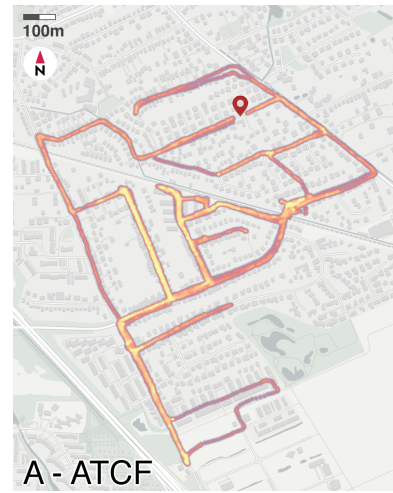

(a)

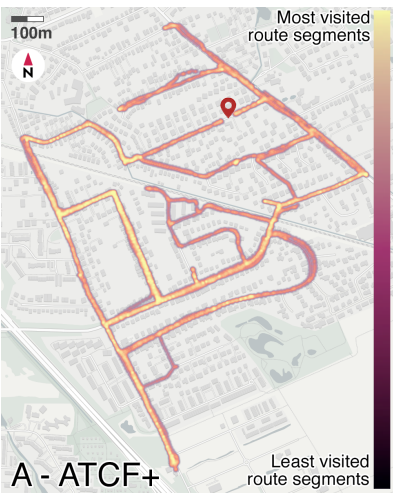

(b)
Figure 5: Heat maps of the uncorrected location data of all participants from the two conditions in study 2. (a) ATCF, (b) ATCF+. A 25m trigger radius causes the gap around the destination. Basemap $\odot$ OpenStreetMap.

no basis for deciding whether to turn left or right. Three participants struggled with this situation turning left towards the start (two participants) or back to where they came from (one participant).

4.5.3 Task Load. Participants scored a numerically slightly lower task load using ATCF (mean $=47.1$, median $=48.3$, sd $=5.8$ ) than with ATCF $+($ mean $=51.7$, median $=56.7, \mathrm{sd}=16.7)$, but the difference was not significant $(\mathrm{t}=-0.68, \mathrm{df}=7.40, \mathrm{p}=0.5185 \mid$ effect size $=0.36, \mathrm{ci}=-0.70-1.41)$.

4.5.4 Orientation. The mean deviation angle when pointing back to the start location was lower with ATCF $\left(\right.$ mean $=19^{\circ}$, median $=$ $\left.12^{\circ} \mathrm{sd}=16^{\circ}\right)$ than with ATCF $+\left(\right.$ mean $=38^{\circ}$, median $=16^{\circ}, \mathrm{sd}=$ $\left.42^{\circ}\right)$. Although this difference was not significant $(\mathrm{t}=-1.12 \mathrm{df}=$ 7.74, $\mathrm{p}=0.295 \mid$ effect size $=0.6, \mathrm{ci}=-0.49-1.66)$, it is interesting to note that the difference in the means is caused by two participants in the ATCF+ condition who had a deviation of $88^{\circ}$ and $107^{\circ}$. All other participants in both groups had a deviation lower than $40^{\circ}$.

4.5.5 Distance. An important feature of visual ATCF navigation is to present the current straight line distance to the destination to the user (see figures 1 and 4). This feature was mistaken by many participants, who are used to TBT navigation, as an indication of progress. TBT navigation usually uses the remaining travel time, remaining distance or both to report progress during the ride. In the case of TBT navigation the remaining travel distance should always decrease as long as one follows the predefined route of the navigation system. With ATCF navigation, however, the straight line distance is not representative of the actual remaining travel distance, as the street network in most cases does not allow for a straight path towards the destination. So whenever one is deviating more than 90 degrees from the target direction, one is actually increasing the straight line distance between the current location and the destination. This is in most cases unavoidable, but can have a severe impact on user confidence.

To test this impact, we calculated how much the distance to the current goal (waypoint or destination) actually increased during the ride of each participant (for instance in the case of a necessary

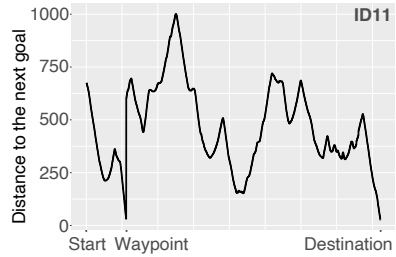

(a)

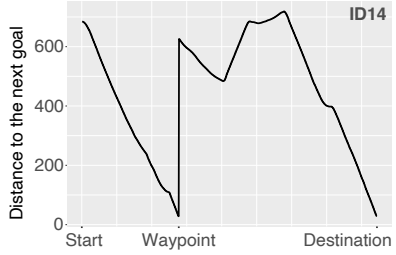

(b)

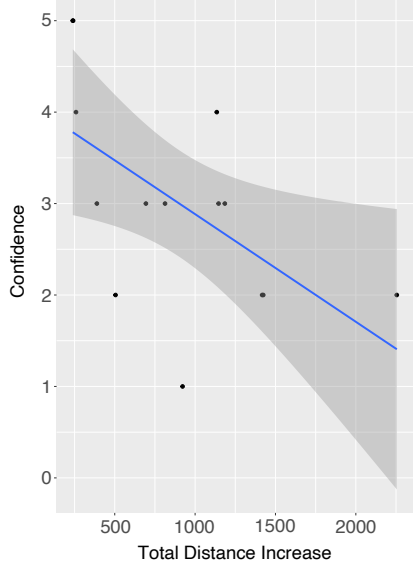

(c)

Figure 6: Distance tracking for ID11, who had a confidence score of 2 (a) and ID14, who had a confidence score of 5 (b). The graph shows the distance to next goal (waypoint or destination) which was indicated on the screen as can be seen in figure 4 a. After reaching the waypoint the distance jumped to indicate the distance to the final destination; (c) The correlation between user confidence (from the questionnaire) and the total increase of the distance to the destination or waypoint on their trip.

detour into the opposite direction of the goal). We summed up all those incidents for each participants which were either caused by the layout of the street network or by errors in decision making by the participants. Figure 6 shows two examples: One of a run without any mistakes (figure 6b) with a total increase of $241 \mathrm{~m}$, and a run where the participant struggled to find the correct way (figure 6a), where all incidents accumulated to a total increase of $2255 \mathrm{~m}$. Through a Pearson's correlation we found that these numbers negatively correlate with the confidence rating $(1=$ not sure $-5=$ sure $)$ that participants gave when asked about how sure they felt during their ride that they would actually reach their destination $(t=-2.44$, $\mathrm{df}=12, \mathrm{p}$-value $=0.03, \mathrm{ci}=-0.85-0.067)$. Figure $6 \mathrm{c}$ shows that their confidence was lower when they experienced a higher total distance increase.

We should think carefully about how to present progress with ATCF navigation. Especially when most people are already familiar with TBT navigation. Some participants told us, that at some point they were actively using the distance indication rather than the arrow to find the correct way, as in their thinking reducing that distance was a valid strategy.

\subsection{Summary}

Our results confirm that indicating that users were still on the right route helped to keep participants from turning around when the arrow was facing in the opposite direction. This confirms H1. Nevertheless, both groups still made a similar number of errors. Participants were able to avoid the most difficult dead ends, but uncovered new ambiguous situations where they would turn into 
the wrong direction due to the lack of information about the surroundings. Also, ATCF+ navigation did not succeed in shortening the routes. Therefore, neither $\mathrm{H} 2$ nor $\mathrm{H} 3$ can be confirmed. One main reason for the lack of improvement in route length is that several participants using ATCF+ took a large detour (see figure 2a, number 3). Some participants using ATCF also started on this route but turned around quite quickly (see figure $5 \mathrm{a}$ ). This highlights an effect of the green background functionality (see figure $4 \mathrm{~b}$ ) as people were reassured after running into the detour that they were still on the right track. This result made us realise that longer routes are not necessarily a bad thing, as long as the participant takes informed decisions, which was one of the main goals of the $\mathrm{ATCF}+$ method.

The most interesting insight concerned the way in which participants relied on the on-screen distance indication (see figure 4a). Some participants told us in the interviews that they did not fully understand the ATCF navigation method until halfway through their ride. So, not only did they sometimes mistake the arrow for a turn-by-turn instruction (supporting similar findings by Pielot et al. [25]), but they also tried to use the distance indication as a basis for navigation - which did not always work out. Thus, participants often travelled further away from the destination which then decreased their confidence of reaching the destination at all. Still, 12 out of 14 participants would choose ATCF over TBT navigation if time constraints were not an issue on their route. This supports findings of Albrecht et al. [1] who found that TBT navigation was preferred when in a hurry, whereas ATCF guidance was preferred for exploration.

All of the results show small to medium effect sizes with large confidence intervals, which means in future work more data is needed to show if we can be sure that the observed effects in this study really exist (according to thresholds presented in [29]). This is also reflected in a post-hoc power analysis using $\mathrm{G}^{*}$ Power for study 2 , which revealed a mean power of 0.21 [9].

\section{DISCUSSION}

Our two studies compared two basic navigation methods (TBT as a highly established one, and ATCF as a novel one whose popularity is currently increasing) with each other (study 1), and further addressed some possible design improvements for the novel ATCF method (study 2).

For purposes of fast and error free navigation, there is little doubt that visual TBT navigation is superior to visual ATCF navigation, at least for users already familiar with TBT. TBT reliably removes uncertainty especially in the case of unforeseeable obstacles and dead ends. In contrast, ATCF provides much more freedom to choose a route while still remaining oriented towards the destination, leading to a potential preference for ATCF for leisure biking. This result extends the findings of Pielot et al. and Albrecht et al. on auditory [1] and tactile [25] ATCF interfaces to visual ATCF interfaces. It shows that visual ATCF navigation has a lot of intuitive appeal for users previously unfamiliar with it, in spite of the associated increased route length and effort.

Our studies were specifically designed in such a way as to create difficult situations for the ATCF navigation method. While related literature suggests that TBT navigation is more efficient than ATCF navigation [1], we present first insights about situations and behaviour that cause these differences in efficiency. We also found indications that visual ATCF interfaces may improve the user's orientation as compared to classical TBT interfaces; this possible effect will need to be confirmed in subsequent studies. Study 2 introduced practical improvements to the design and functionality of visual ATCF interfaces. The improved interface version appeared to help participants avoid the error of turning round when the arrow was pointing in the opposite direction of travel. Also, study 2 highlighted additional challenges. In the following three sections we address our main insights in detail.

\subsection{Cycling In the Opposite Direction to the Arrow}

Ideally the shortest path towards a destination is a straight line between the user and where they want to go. If that was always the case, one would always find the shortest path using ATCF navigation, for instance on an open field with no restraining streets or obstacles.

In a street network, the straight line to the goal is typically constrained by the roads and blocked by buildings, parks, bodies of water and the like. Users of ATCF navigation will therefore often have to deviate from the ideal direction of travel, sometimes so much so that they're actually heading towards the opposite direction for a while. As a consequence, we saw participants turning around (Route A) or finding alternative but very bike-unfriendly paths (Route B) to escape this kind of situation. This is related to the lack of knowledge participants had about the route and surrounding street network, as aptly expressed by one participant in study 2 (ATCF condition): "Is there still a path back there, or am I going in the wrong direction for 5 kilometres hoping that there will be a path?". These results support findings by Albrecht et al. where long stretches without anywhere to turn caused uncertainty among participants [1]. Similar to Albrecht et al. [1], we did not show participants a map of the area or the position of the waypoint or destination. The mini-map we introduced with the ATCF+ version did not seem to make much of a difference. One participant told us that they tried to click the map to enlarge it which didn't work. They suggested a full screen map with a small compass needle for ATCF navigation, which could be a worthwhile concept to test in future studies.

\subsection{Route Progression}

Cycling in the opposite direction to the arrow particularly affected participants' sense of making progress towards the destination. During the interviews of study 2 participants told us that they often kept track of the distance indicated at the top of the screen (see figure 4a). Many of them used it as a way to check their progress and evaluate their navigation decisions. One participant even solely relied on the distance indication because they had problems understanding the functionality of the compass. The problem with this is that relying on the distance can be very counter-intuitive when using ATCF navigation systems, as the straight line distance displayed on the screen might rise even though one is on the fastest path (e.g., at number 2 in figure 2). 
Turn-by-turn navigation systems, in contrast, clearly indicate progress relative to the destination. Their main feature is to convert the task of minimising the distance between the user and the destination into following a set of instructions. The route length that is presented to the user directly corresponds to the length of the route when following the instructions. This representation is more accurate and reliable when it comes to representing progress. The fact that this behaviour was not observed or reported in study 1 could mean that the design of the remaining distance indicator was mainly responsible for the confusion (compare figure 1 and figure 4) - i.e., it may be important that the distance is provided directly within the arrow rather than separately. In any case, improving user confidence concerning progress towards the goal while using ATCF navigation is an important point to consider for improving ATCF user interfaces. As the total length of the journey is not known in advance, this is difficult to assess. One way could be to estimate the average route length based on possible paths that are likely to be taken by the user.

\subsection{Cyclists Go Everywhere}

An observation that could make such an estimation difficult is that cyclists will find many different paths. Our participants were not only willing to go to great lengths to follow the direction indicated by the ATCF navigation system, but also found creative pathways through inaccessible terrain or private property. This is potentially very important when thinking about how navigation systems for cyclists should differ from navigation systems for cars. A navigation system for cyclists should not limit its knowledge base to the official street network but allow for and react accordingly to off-road riding behaviour.

\section{LIMITATIONS}

Many participants reported that they had problems understanding the underlying ATCF navigation principles at first glance. While all of them were used to TBT navigation systems, a steep learning curve was required for the ATCF interface. In future studies, it would be useful to provide a practical training phase before the study for ATCF and especially ATCF+ (with its extended features), so as to make sure that participants are fully comfortable with the functionalities and information provided in the interface. Since none of the participants in our studies had previous experience with ATCF navigation, the results are consistent throughout our samples in that regard, but the comparison to TBT is clearly influenced by the difference in familiarity with the navigation method.

Furthermore, the results of this study are influenced by the cycling infrastructure in Germany. In other countries with different cycle paths and infrastructure, a visual ATCF navigation system might be used in different ways than that which we observed. ATCF navigation also relies heavily on the street layout. Not only will using it in urban or rural areas make a difference, but also different cities with a more grid-like street layout (like many large US cities) or more unstructured ones (like most European cities) will probably influence the way people use ATCF navigation. Finally, the comparison favours TBT over ATCF as both studies include a "Go from A to B" type navigation task, which TBT navigation is very well suited for. A more open task like the one used in Pielot et al. [25] could paint a very different picture when comparing both navigation methods.

\section{FUTURE WORK}

Visual ATCF navigation is already used in consumer navigation systems. However, there it is usually an alternative to TBT navigation. We envision multiple approaches that combine some of the aspects of ATCF and TBT navigation. One approach could for example try to insert multiple waypoints on the route while minimising the deviation angle for these subsections. This could eliminate situations where the arrow points in the opposite direction of travel. The effect of such a method on orientation and spatial knowledge acquisition would need to be tested. One participant suggested to combine the map from TBT navigation with a notion of the general direction. Future work could test different visualisations of this concept and for example investigate how route suggestions could look like for ATCF navigation. In their current design, most visual ATCF navigation methods do not communicate a route's efficiency or appropriateness in different situations (e.g. informing users about potential dead ends that they are likely to run into), which leaves some potential for frustration. They might be very well suited for open tasks like exploring a new place without time restrictions as in Pielot et al. [25], but less so if you urgently need to reach a specific destination.

Future work could investigate how ATCF navigation systems can give users a clear idea of how well suited they will be for the given task and when or if they should offer assistance.

\section{CONCLUSION}

Our results show that the route design effectively introduced a challenging situation for participants using the standard ATCF navigation method. Participants had problems reaching their destination without errors, detours or creative wayfinding behaviour (e.g. carry their bike up a staircase, riding through private property). Our ATCF+ navigation interface, using coloured cues, successfully assisted participants in some of these situations, alongside highlighting further unanticipated effects such as over-reliance on the distance information. This increases our insights on ATCF navigation. In agreement with related literature $[1,25]$, we find that ATCF navigation is less efficient than TBT navigation (in terms of route length and navigation errors) and users prefer TBT navigation in situations where they need error free, fast, reliable navigation. Nevertheless, many users would prefer ATCF over TBT navigation for leisure biking.

While ATCF navigation is at the moment exclusive to bicycle navigation, it might only be a matter of time until it finds its way into other more mainstream navigation systems. With more research on this topic HCI researchers will be able to successfully guide decisions in this domain and increasingly understand the advantages and disadvantages of ATCF navigation.

\section{ACKNOWLEDGMENTS}

We would like to thank the participants of our study for their time. This work is part of the Volkswagen Foundation through a Lichtenberg Professorship. 


\section{REFERENCES}

[1] Robert Albrecht, Riitta Väänänen, and Tapio Lokki. 2016. Guided by Music: Pedestrian and Cyclist Navigation with Route and Beacon Guidance. Personal Ubiquitous Comput. 20, 1 (Feb. 2016), 121-145. https://doi.org/10.1007/s00779016-0906-z

[2] Beeline. 2019. Beeline Velo. Website. Retrieved August, 252019 from https: //beeline.co/pages/beeline-velo.

[3] Jens Blauert. 1997. Spatial hearing: the psychophysics of human sound localization. MIT press, Harvard, MA, USA.

[4] Blubel. 2019. Blubel. Website. Retrieved July, 242019 from https://blubel.co/.

[5] Alexandru Dancu, Zlatko Franjcic, and Morten Fjeld. 2014. Smart Flashlight: Map Navigation Using a Bike-mounted Projector. In Proceedings of the 32Nd Annual ACM Conference on Human Factors in Computing Systems (Toronto, Ontario, Canada) (CHI '14). ACM, New York, NY, USA, 3627-3630. https://doi.org/10. $1145 / 2556288.2557289$

[6] Alexandru Dancu, Velko Vechev, Adviye Ayça Ünlüer, Simon Nilson, Oscar Nygren, Simon Eliasson, Jean-Elie Barjonet, Joe Marshall, and Morten Fjeld. 2015. Gesture Bike: Examining Projection Surfaces and Turn Signal Systems for Urban Cycling. In Proceedings of the 2015 International Conference on Interactive Tabletops \& Surfaces (Madeira, Portugal) (ITS '15). ACM, New York, NY, USA, 151-159. https://doi.org/10.1145/2817721.2817748

[7] Dick de Waard, Frank Westerhuis, Danielle Joling, Stella Weiland, Ronja Stadtbäumer, and Leonie Kaltofen. 2017. Visual map and instruction-based bicycle navigation: a comparison of effects on behaviour. Ergonomics 60, 9 (2017), 12831296. https://doi.org/10.1080/00140139.2017.1282628 PMID: 28092224

[8] Matt Duckham and Lars Kulik. 2003. "Simplest" Paths: Automated Route Selection for Navigation. In Spatial Information Theory. Foundations of Geographic Information Science, Walter Kuhn, Michael F. Worboys, and Sabine Timpf (Eds.) Springer Berlin Heidelberg, Berlin, Heidelberg, 169-185.

[9] Franz Faul, Edgar Erdfelder, Albert-Georg Lang, and Buchner AG. 2007. G*Power 3: A flexible statistical power analysis program for the social, behavioral, and biomedical sciences. Behavior Research Methods Instruments \& Computers 39 (01 2007), 175-191.

[10] Reginald G. Golledge. 1995. Path selection and route preference in human navigation: A progress report. In Spatial Information Theory A Theoretical Basis for GIS, Andrew U. Frank and Werner Kuhn (Eds.). Springer Berlin Heidelberg, Berlin, Heidelberg, 207-222.

[11] Weixi Gu, Zimu Zhou, Yuxun Zhou, Han Zou, Yunxin Liu, Costas J. Spanos, and Lin Zhang. 2017. BikeMate: Bike Riding Behavior Monitoring with Smartphones. In Proceedings of the 14th EAI International Conference on Mobile and Ubiquitous Systems: Computing, Networking and Services (Melbourne, VIC, Australia) (MobiQuitous 2017). ACM, New York, NY, USA, 313-322. https://doi.org/10.1145/ 3144457.3144462

[12] Florian Heller and Johannes Schöning. 2018. NavigaTone: Seamlessly Embedding Navigation Cues in Mobile Music Listening. In Proceedings of the $2018 \mathrm{CHI}$ Conference on Human Factors in Computing Systems (Montreal QC, Canada) (CHI '18). Association for Computing Machinery, New York, NY, USA, 1-7. https://doi.org/10.1145/3173574.3174211

[13] Hartwig H Hochmair. 2005. Investigating the Effectiveness of the Least-Angle Strategy for Wayfinding in Unknown Street Networks. Environment and Planning B: Planning and Design 32, 5 (2005), 673-691. https://doi.org/10.1068/b31160

[14] Hartwig Hochmair H. 2000. 'Least Angle' Heuristic: Consequences of errors during navigation (abstract).

[15] Hartwig Hochmair $\mathrm{H}$ and Andrew Frank. 2000. Influence of estimation errors on wayfinding-decisions in unknown street networks - Analyzing the leastangle strategy. Spatial Cognition and Computation 2 (01 2000), 283-313. https: //doi.org/10.1023/A:1015566423907

[16] Simon Holland, David R. Morse, and Henrik Gedenryd. 2002. AudioGPS: Spatial Audio Navigation with a Minimal Attention Interface. Personal Ubiquitous Comput. 6, 4 (Jan. 2002), 253-259. https://doi.org/10.1007/s007790200025

[17] Isaac Johnson, J. Henderson, C. Perry, Johannes Schöning, and Brent Hecht. 2017. Beautiful... but at What Cost? An Examination of Externalities in Geographic Vehicle Routing. Proc. ACM Interact. Mob. Wearable Ubiquitous Technol. 1, 2, Article 15 (June 2017), 21 pages. https://doi.org/10.1145/3090080

[18] Allen Yilun Lin, Kate Kuehl, Johannes Schöning, and Brent Hecht. 2017. Understanding "Death by GPS": A Systematic Study of Catastrophic Incidents Associated with Personal Navigation Technologies. In Proceedings of the 2017 CHI Conference on Human Factors in Computing Systems (Denver, Colorado, USA)
(CHI '17). Association for Computing Machinery, New York, NY, USA, 1154-1166. https://doi.org/10.1145/3025453.3025737

[19] Jack M. Loomis, Reginald G. Golledge, and Roberta L. Klatzky. 1998. Navigation System for the Blind: Auditory Display Modes and Guidance. Presence: Teleoperators and Virtual Environments 7, 2 (1998), 193-203. https://doi.org/10.1162/ 105474698565677 arXiv:https://doi.org/10.1162/105474698565677

[20] Andrii Matviienko, Swamy Ananthanarayan, Abdallah El Ali, Wilko Heuten, and Susanne Boll. 2019. NaviBike: Comparing Unimodal Navigation Cues for Child Cyclists. In Proceedings of the 2019 CHI Conference on Human Factors in Computing Systems (Glasgow, Scotland Uk) (CHI '19). ACM, New York, NY, USA, Article 620, 12 pages. https://doi.org/10.1145/3290605.3300850

[21] NASA. 2019. NASA TLX application. Website. Retrieved January, 312019 from https:/humansystems.arc.nasa.gov/groups/tlx/tlxapp.php.

[22] openrouteservice. 2019. openrouteservice. Website. Retrieved August, 252019 from https://maps.openrouteservice.org/.

[23] Martin Pielot, Benjamin Poppinga, and Susanne Boll. 2009. Understanding tourists on a bicycle trip" in the wild. , 19 pages.

[24] Martin Pielot, Benjamin Poppinga, Wilko Heuten, and Susanne Boll. 2011. A Tactile Compass for Eyes-Free Pedestrian Navigation. In Human-Computer Interaction - INTERACT 2011, Pedro Campos, Nicholas Graham, Joaquim Jorge, Nuno Nunes, Philippe Palanque, and Marco Winckler (Eds.). Springer Berlin Heidelberg, Berlin, Heidelberg, 640-656.

[25] Martin Pielot, Benjamin Poppinga, Wilko Heuten, and Susanne Boll. 2012. Tacticycle: Supporting Exploratory Bicycle Trips. In Proceedings of the 14th International Conference on Human-computer Interaction with Mobile Devices and Services (San Francisco, California, USA) (MobileHCI '12). ACM, New York, NY, USA, 369-378. https://doi.org/10.1145/2371574.2371631

[26] Benjamin Poppinga, Martin Pielot, and Susanne Boll. 2009. Tacticycle: A Tactile Display for Supporting Tourists on a Bicycle Trip. In Proceedings of the 11th International Conference on Human-Computer Interaction with Mobile Devices and Services (Bonn, Germany) (MobileHCI '09). ACM, New York, NY, USA, Article 41, 4 pages. https://doi.org/10.1145/1613858.1613911

[27] Manoj Prasad, Paul Taele, Daniel Goldberg, and Tracy A. Hammond. 2014. HaptiMoto: Turn-by-turn Haptic Route Guidance Interface for Motorcyclists. In Proceedings of the SIGCHI Conference on Human Factors in Computing Systems (Toronto, Ontario, Canada) (CHI '14). ACM, New York, NY, USA, 3597-3606. https://doi.org/10.1145/2556288.2557404

[28] Kai-Florian Richter, Alexander Klippel, and Christian Freksa. 2004. Shortest, fastest,-but what next? a different approach to route directions. , 205-217 pages.

[29] Judy Robertson and Maurits Kaptein. 2016. Modern Statistical Methods for HCI (1st ed.). Springer Publishing Company, Incorporated, Switzerland.

[30] Simon Robinson, Matt Jones, Parisa Eslambolchilar, Roderick Murray-Smith, and Mads Lindborg. 2010. "I Did It My Way": Moving Away from the Tyranny of Turn-by-turn Pedestrian Navigation. In Proceedings of the 12th International Conference on Human Computer Interaction with Mobile Devices and Services (Lisbon, Portugal) (MobileHCI '10). ACM, New York, NY, USA, 341-344. https: //doi.org/10.1145/1851600.1851660

[31] Steven M. Selbst, David Alexander, and Richard Ruddy. 1987. Bicycle-Related Injuries. JAMA Pediatrics 141, 2 (02 1987), 140-144. https://doi.org/10.1001/ archpedi.1987.04460020030021

[32] SmartHalo. 2019. SmartHalo 2. Website. Retrieved August, 252019 from https://www.youtube.com/watch?v=ZNDrGw7Bxjw.

[33] Haska Steltenpohl and Anders Bouwer. 2013. Vibrobelt: Tactile Navigation Support for Cyclists. In Proceedings of the 2013 International Conference on Intelligent User Interfaces (Santa Monica, California, USA) (IUI '13). ACM, New York, NY, USA, 417-426. https://doi.org/10.1145/2449396.2449450

[34] Hung-Yu Tseng, Rong-Hao Liang, Liwei Chan, and Bing-Yu Chen. 2015. LEaD: Utilizing Light Movement As Peripheral Visual Guidance for Scooter Navigation. In Proceedings of the 17th International Conference on Human-Computer Interaction with Mobile Devices and Services (Copenhagen, Denmark) (MobileHCI '15). ACM, New York, NY, USA, 323-326. https://doi.org/10.1145/2785830.2785831

[35] Shanjiang Zhu and David Levinson. 2015. Do People Use the Shortest Path? An Empirical Test of Wardrop's First Principle. PLOS ONE 10, 8 (08 2015), 1-18. https://doi.org/10.1371/journal.pone.0134322

[36] Matthijs Zwinderman, Tanya Zavialova, Daniel Tetteroo, and Paul Lehouck. 2011. Oh Music, Where Art Thou?. In Proceedings of the 13th International Conference on Human Computer Interaction with Mobile Devices and Services (Stockholm, Sweden) (MobileHCI '11). ACM, New York, NY, USA, 533-538. https: //doi.org/10.1145/2037373.2037456 